\title{
Analysis of the relationship between insomnia and adult chronic diseases with regard to working conditions
}

\author{
WERONIKA WOLIŃSKA ${ }^{A-F}$, HALINA BRZEŹNIAK ${ }^{B, F}$, BOŻENA MROCZEKA, D, E, G \\ ORCID ID: 0000-0002-3358-8280 ORCID ID: 0000-0002-3883-4145 ORCID ID: 0000-0002-6745-4942
}

Department of Humanities in Medicine, Pomeranian Medical University, Szczecin, Poland

A - Study Design, B - Data Collection, C - Statistical Analysis, D - Data Interpretation, E - Manuscript Preparation, F - Literature

Search, G - Funds Collection

Summary Background. Sleep is one of the most important physiological needs of man. It ensures health, regeneration and the concentration necessary to fulfil professional roles.

Objectives. The aim of this study was to determine the relationship between insomnia and selected adult chronic diseases, taking into account working conditions and employment status.

Material and methods. The study involved 597 individuals, including 360 (60.3\%) women and $237(39.7 \%)$ men, as well as 396 (66.33\%) employed and $201(33.67 \%)$ unemployed subjects. The instruments used in the study were the Athens Insomnia Scale (AIS) and a self-developed sociodemographic questionnaire.

Results. The most common disease was hypertension $(116 ; 19.43 \%)$. There was a statistically significant relationship between hypertension and employment status $(p \leq 0.001)$, as well as between asthma/COPD and employment status $(p=0.012)$. COPD was more prevalent in unemployed subjects. Every fourth person with hypertension suffered from insomnia. Insomnia was observed in every fifth person working shifts, and one-third of them was at risk of insomnia.

Conclusions. Unemployed respondents more often suffered from various diseases. The age factor and working and living conditions (contract work, shift work, unemployment) are factors contributing to the occurrence or worsening of insomnia and chronic diseases. Insomnia and a lack of depth and quality of sleep can be due to the level of professional and life stress, working and living conditions, social instability and chronic diseases.

Key words: sleep initiation and maintenance disorders, chronic disease, adult.

Wolińska W, Brzeźniak H, Mroczek B. Analysis of the relationship between insomnia and adult chronic diseases with regard to working conditions. Fam Med Prim Care Rev 2020; 22(3): 228-234, doi: https://doi.org/10.5114/fmpcr.2020.98251.

\section{Background}

Sleep is one of the most important physiological needs of man. It ensures health, regeneration and the concentration necessary to fulfil professional roles [1]. According to the Diagnostic and Statistical Manual of Mental Disorders, Fifth Edition (DSM-5), insomnia is diagnosed when one or more symptoms occur, such as difficulty falling asleep, difficulty in maintaining sleep continuity (frequent waking up at night and being unable to fall asleep again) and waking up early in the morning and being unable to fall asleep. These symptoms must occur for at least three nights a week and last for at least three months [2].

Insomnia is determined by many factors, among them chronic diseases, age and gender. Studies have shown that insomnia is related to cardiovascular and metabolic disorders, such as coronary thrombosis, stroke, hypertension, diabetes, obesity, atherosclerosis, heart failure, arrhythmia and others [3]. Insufficient time of sleep and its low quality can contribute to hypertension [4]. Changes in sleep structure and phases are more often observed in people suffering from hypertension than in those who have not been diagnosed with this disease. Hypertensive patients more often wake up in the middle of the night, and sleep does not give them the opportunity to regenerate and rest [5].

The circadian rhythm of sleep plays a significant role in diabetes [6]. The following changes in sleep phases were observed in a group of people with type 2 diabetes: a decreased amount of non-rapid eye movement (NREM) sleep, a larger amount of rapid eye movement (REM) sleep, as well as more frequent awakening during the night with an inability to fall asleep again [7]. In the course of chronic insomnia, reduced sleep is manifested by glucose intolerance in the body, which in the long term can lead to a decrease in the amount of insulin in the pancreas [8].

Thyroid diseases also affect human sleep. Both hypothyroidism and hyperthyroidism cause difficulty in falling asleep and maintaining sleep, as well as delayed or early slow-wave sleep $[9,10]$. What is more, intravenous administration of thyrotropin-releasing hormone (TRH) can affect some of the sleep parameters: it can shorten sleep time, cause daytime sleepiness and extend nocturnal awake times [11]

\section{Objectives}

The aim of this study was to determine the relationship between insomnia and selected adult chronic diseases, taking into account working conditions and employment status.

Hypotheses:

1) chronic diseases are more common in unemployed people,

2) insomnia is often related to chronic diseases,

3) sleep problems are related to employment status.

\section{Material and methods}

\section{Study design}

This was a cross-sectional retrospective study. 


\begin{tabular}{|c|c|c|c|c|}
\hline Variable & & $\begin{array}{l}\text { Total } \\
n(\%)\end{array}$ & $\begin{array}{l}\text { Employed } \\
n(\%)\end{array}$ & $\begin{array}{l}\text { Unemployed } \\
n(\%)\end{array}$ \\
\hline Gender & $\begin{array}{l}\text { women } \\
\text { men }\end{array}$ & $\begin{array}{l}360(60.3) \\
237(39.7)\end{array}$ & $\begin{array}{l}230(63.89) \\
166(70.04) \\
\end{array}$ & $\begin{array}{l}130(36.11) \\
71(29.96)\end{array}$ \\
\hline Age & $\begin{array}{l}\text { up to } 25 \text { years } \\
26-35 \\
36-45 \\
46-55 \\
\text { over } 56 \text { years }\end{array}$ & $\begin{array}{l}171(28.64) \\
157(26.3) \\
80(13.4) \\
93(15.58) \\
96(16.08) \\
\end{array}$ & $\begin{array}{l}99(57.9) \\
128(81.53) \\
69(86.25) \\
72(77.42) \\
28(29.17) \\
\end{array}$ & $\begin{array}{l}72(42.1) \\
29(18.47) \\
11(13.75) \\
21(22.58) \\
68(70.83) \\
\end{array}$ \\
\hline BMI & $\begin{array}{l}\text { underweight } \\
\text { normal weight } \\
\text { overweight } \\
\text { obesity }\end{array}$ & $\begin{array}{l}24(4.02) \\
312(52.26) \\
210(35.18) \\
51(8.54) \\
\end{array}$ & $\begin{array}{l}16(66.67) \\
219(70.19) \\
132(62.86) \\
29(56.86) \\
\end{array}$ & $\begin{array}{l}8(33.33) \\
93(29.81) \\
78(37.14) \\
22(43.14) \\
\end{array}$ \\
\hline \multirow[t]{5}{*}{ Form of employment } & $\begin{array}{l}\text { employment contract } \\
\text { yes } \\
\text { no }\end{array}$ & - & $\begin{array}{l}277(69.95) \\
119(30.05)\end{array}$ & - \\
\hline & $\begin{array}{l}\text { freelance agreement } \\
\text { yes } \\
\text { no }\end{array}$ & - & $\begin{array}{l}73(18.43) \\
323(81.57)\end{array}$ & - \\
\hline & $\begin{array}{l}\text { contract for a specific } \\
\text { task } \\
\text { yes } \\
\text { no }\end{array}$ & - & $\begin{array}{l}5(1.26) \\
391(98.74)\end{array}$ & - \\
\hline & $\begin{array}{l}\text { contract } \\
\text { yes } \\
\text { no }\end{array}$ & - & $\begin{array}{l}20(5.05) \\
376(94.95)\end{array}$ & - \\
\hline & $\begin{array}{l}\text { other } \\
\text { yes } \\
\text { no }\end{array}$ & - & $\begin{array}{l}34(8.61) \\
361(91.39)\end{array}$ & - \\
\hline Years worked & $\begin{array}{l}\text { one year or less } \\
1-3 \text { years } \\
3-8 \text { years } \\
9-18 \text { years } \\
\text { over } 18 \text { years }\end{array}$ & - & $\begin{array}{l}103(26.01) \\
92(23.23) \\
84(21.21) \\
64(16.16) \\
53(13.38)\end{array}$ & $\begin{array}{l}- \\
- \\
- \\
- \\
-\end{array}$ \\
\hline Shift work & $\begin{array}{l}\text { yes } \\
\text { no } \\
\text { not given }\end{array}$ & $\begin{array}{l}201(33.67) \\
385(64.49) \\
11(1.84) \\
\end{array}$ & $\begin{array}{l}137(68.16) \\
259(67.27) \\
- \\
\end{array}$ & $\begin{array}{l}64(31.84) \\
126(32.73) \\
11(100) \\
\end{array}$ \\
\hline $\begin{array}{l}\text { Self-assessed financial } \\
\text { standing }\end{array}$ & $\begin{array}{l}\text { very good } \\
\text { good } \\
\text { satisfactory } \\
\text { bad }\end{array}$ & $\begin{array}{l}62(10.39) \\
299(50.08) \\
208(34.84) \\
28(4.69)\end{array}$ & $\begin{array}{l}51(82.26) \\
208(69.57) \\
125(60.1) \\
12(42.86)\end{array}$ & $\begin{array}{l}11(17.74) \\
91(30.43) \\
83(39.9) \\
16(57.14)\end{array}$ \\
\hline
\end{tabular}

\section{Setting and ethical consideration}

The study was conducted in Poland. The criteria for inclusion in the study were being 18 to 65 years of age, residing in West Pomeranian Voivodeship and consent to participate in the study. Respondents were selected using quota sampling based on gender, place of residence and employment status.

The project was approved by the Bioethical Commission of the Pomeranian Medical University, Szczecin (KB-0012/63/16).

\section{Participants}

The study involved 597 individuals, including 360 (60.3\%) women and 237 (39.7\%) men. $396(66.33 \%)$ respondents were employed, and 201 (33.67\%) were unemployed. The majority of the participants were up to 25 years of age ( $n=171,28.64 \%)$, most of whom were employed ( $n=99,57.9 \%)$. The predominant form of employment was an employment contract ( $n=277,69.95 \%$ ). In the case of over one-fourth of the participants, the number of years worked was one year or less $(n=103,26.01 \%)$ (Table 1$)$.

\section{Data sources}

This survey-based study was performed using the Athens Insomnia Scale (AIS) and a self-developed sociodemographic questionnaire.
The AIS consists of eight questions concerning the frequency and severity of insomnia symptoms. A given symptom should be marked if it occurred at least three times a week for at least a month. Responses are rated on a four-point scale and scored $0-3$. The AIS total scores are interpreted as follows: $<5$ points - no insomnia, 6-10 points - borderline insomnia, $>10$ points - insomnia.

\section{Statistical analysis}

Statistical analysis was performed using Statistica 13 . The normality of the distribution was assessed by the Shapiro-Wilk $\mathrm{W}$ test. The incidence of insomnia with regard to the adopted variables was determined using the chi-square test for independence. Statistical significance was set at $p \leq 0.05$.

\section{Results}

Hypothesis 1: Chronic diseases are more common in unemployed people

The majority of the respondents had hypertension (116; $19.43 \%)$ - the percentage of employed and unemployed sub- 
jects was the same - 58 (50\%). There was a statistically significant relationship between hypertension and employment status $(p \leq 0.001)$ and between asthma/COPD and employment status ( $p=0.012)$ - COPD was more common in unemployed respondents ( $n=19 ; 52.78 \%$ ) (Table 2). 23 (3.85\%) respondents had other cardiovascular diseases: varicose veins, tachycardia, valve diseases, low blood pressure, anaemia, arrhythmia, coronary thrombosis, neuropathy. 35 (5.86\%) respondents had digestive diseases. The multimorbidity rate was
0.52 . The person with the largest number of diseases indicated five disease entities.

Other cardiovascular diseases were statistically significantly related to gender (they were significantly more common in women) ( $n=20, p=0.007)$ and to hyperthyroidism ( $n=32, p \leq$ 0.001). Chronic diseases were mainly noted in people over 56 years of age. Diabetes was statistically significantly related to BMI (it was more common in overweight individuals) ( $n=23$, $p \leq 0.001)$ and to atherosclerosis $(n=12, p=0.035$ ) (Table 3 ).

\begin{tabular}{|c|c|c|c|c|c|}
\hline \multicolumn{2}{|l|}{ Variable } & $\begin{array}{l}\text { Total } \\
n(\%)\end{array}$ & $\begin{array}{l}\text { Employed } \\
n(\%)\end{array}$ & $\begin{array}{l}\text { Unemployed } \\
n(\%)\end{array}$ & $\begin{array}{l}\mathrm{Chi}^{2} \\
p \\
{ }^{*} \mathrm{Fi}\end{array}$ \\
\hline \multirow[t]{7}{*}{ Chronic diseases } & $\begin{array}{l}\text { diabetes } \\
\text { yes } \\
\text { no }\end{array}$ & $\begin{array}{l}42(7.04) \\
555(92.96)\end{array}$ & $\begin{array}{l}19(45.24) \\
377(67.93)\end{array}$ & $\begin{array}{l}23(54.76) \\
178(32.07)\end{array}$ & $\begin{array}{l}9.000 \\
\mathbf{0 . 0 0 3} \\
*_{0} 0.122\end{array}$ \\
\hline & $\begin{array}{l}\text { hypertension } \\
\text { yes } \\
\text { no }\end{array}$ & $\begin{array}{l}116(19.43) \\
481(80.57)\end{array}$ & $\begin{array}{l}58(50) \\
338(70.27)\end{array}$ & $\begin{array}{l}58(50) \\
143(29.73)\end{array}$ & $\begin{array}{l}17.195 \\
\leq 0.001 \\
* 0.169\end{array}$ \\
\hline & $\begin{array}{l}\text { asthma/COPD } \\
\text { yes } \\
\text { no }\end{array}$ & $\begin{array}{l}36(6.04) \\
560(93.96)\end{array}$ & $\begin{array}{l}17(47.22) \\
378(67.5) \\
\end{array}$ & $\begin{array}{l}19(52.78) \\
182(32.5)\end{array}$ & $\begin{array}{l}6.222 \\
0.012 \\
* 0.102\end{array}$ \\
\hline & $\begin{array}{l}\text { atherosclerosis } \\
\text { yes } \\
\text { no }\end{array}$ & $\begin{array}{l}22(3.69) \\
575(96.31)\end{array}$ & $\begin{array}{l}3(13.64) \\
393(68.35)\end{array}$ & $\begin{array}{l}19(86.36) \\
182(31.65)\end{array}$ & $\begin{array}{l}28.400 \\
\leq 0.001 \\
* 0.218\end{array}$ \\
\hline & $\begin{array}{l}\text { other cardiovascular diseases } \\
\text { yes } \\
\text { no }\end{array}$ & $\begin{array}{l}23(3.85) \\
574(96.15)\end{array}$ & $\begin{array}{l}10(43.48) \\
386(67.25)\end{array}$ & $\begin{array}{l}13(56.52) \\
188(32.75) \\
\end{array}$ & $\begin{array}{l}5.594 \\
\mathbf{0 . 0 1 8} \\
* 0.096 \\
\end{array}$ \\
\hline & $\begin{array}{l}\text { hyperthyroidism } \\
\text { yes } \\
\text { no }\end{array}$ & $\begin{array}{l}37(6.2) \\
560(93.8)\end{array}$ & $\begin{array}{l}25(67.57) \\
371(66.25)\end{array}$ & $\begin{array}{l}12(32.43) \\
189(33.75)\end{array}$ & $\begin{array}{l}0.269 \\
0.869 \\
-\end{array}$ \\
\hline & $\begin{array}{l}\text { digestive diseases } \\
\text { yes } \\
\text { no }\end{array}$ & $\begin{array}{l}35(5.86) \\
562(94.14)\end{array}$ & $\begin{array}{l}13(37.14) \\
188(33.45)\end{array}$ & $\begin{array}{l}22(62.86) \\
374(66.55)\end{array}$ & $\begin{array}{l}0.200 \\
0.653 \\
-\end{array}$ \\
\hline
\end{tabular}

* Fi - Fi coefficient.

\begin{tabular}{|c|c|c|c|c|c|c|c|c|}
\hline Variable & $\begin{array}{l}\mathrm{Chi}^{2} \\
p \\
* \mathrm{~V} / \\
* * \mathrm{Fi}\end{array}$ & Diabetes & $\begin{array}{l}\text { Hyperten- } \\
\text { sion }\end{array}$ & $\begin{array}{l}\text { Asthma/ } \\
\text { /COPD }\end{array}$ & $\begin{array}{l}\text { Atheroscle- } \\
\text { rosis }\end{array}$ & $\begin{array}{l}\text { Other car- } \\
\text { diovascular } \\
\text { diseases }\end{array}$ & $\begin{array}{l}\text { Hyperthy- } \\
\text { roidism }\end{array}$ & $\begin{array}{l}\text { Digestive } \\
\text { diseases }\end{array}$ \\
\hline $\begin{array}{l}\text { Gender } \\
\text { women } \\
\text { men }\end{array}$ & $\begin{array}{l}\mathrm{Chi}^{2} \\
p \\
* \mathrm{~V} / \\
* * \mathrm{Fi}\end{array}$ & $\begin{array}{l}3.035 \\
0.081 \\
-\end{array}$ & $\begin{array}{l}0.169 \\
0.680 \\
-\end{array}$ & $\begin{array}{l}3.413 \\
0.057 \\
-\end{array}$ & $\begin{array}{l}1.012 \\
0.314 \\
-\end{array}$ & $\begin{array}{l}7.099 \\
\mathbf{0 . 0 0 7} \\
* 0.108\end{array}$ & $\begin{array}{l}11.297 \\
0.000 \\
* 0.136\end{array}$ & $\begin{array}{l}0.101 \\
0.750 \\
-\end{array}$ \\
\hline $\begin{array}{l}\text { Age } \\
\text { up to } 25 \text { years } \\
26-35 \\
36-45 \\
46-55 \\
\text { over } 56 \text { years }\end{array}$ & $\begin{array}{l}\mathrm{Chi}^{2} \\
p \\
* \mathrm{~V} / \\
* * \mathrm{Fi}\end{array}$ & \begin{tabular}{|l}
65.243 \\
$\mathbf{0 . 0 0 0}$ \\
$* 0.330$
\end{tabular} & $\begin{array}{l}202.403 \\
0.000 \\
* 0.582\end{array}$ & \begin{tabular}{|l|}
12.490 \\
$\mathbf{0 . 0 1 4}$ \\
$* 0.144$
\end{tabular} & $\begin{array}{l}60.216 \\
0.000 \\
* 0.317\end{array}$ & $\begin{array}{l}21.436 \\
0.000 \\
* 0.189\end{array}$ & $\begin{array}{l}6.452 \\
0.167 \\
-\end{array}$ & $\begin{array}{l}3.183 \\
0.527 \\
-\end{array}$ \\
\hline $\begin{array}{l}\text { BMI } \\
\text { underweight } \\
\text { normal weight } \\
\text { overweight } \\
\text { obesity }\end{array}$ & $\begin{array}{l}\mathrm{Chi}^{2} \\
p \\
* \mathrm{~V} / \\
* * \mathrm{Fi}\end{array}$ & \begin{tabular}{|l}
32.750 \\
$\mathbf{0 . 0 0 0}$ \\
$* 0.234$
\end{tabular} & $\begin{array}{l}75.707 \\
0.000 \\
* 0.356\end{array}$ & $\begin{array}{l}5.934 \\
0.114 \\
-\end{array}$ & $\begin{array}{l}8.568 \\
0.035 \\
* 0.119\end{array}$ & $\begin{array}{l}1.057 \\
0.787 \\
-\end{array}$ & $\begin{array}{l}6.655 \\
0.083 \\
-\end{array}$ & $\begin{array}{l}1.038 \\
0.791 \\
-\end{array}$ \\
\hline Form of employment & $\begin{array}{l}\mathrm{Chi}^{2} \\
p \\
* \mathrm{~V} / \\
* * \mathrm{Fi}\end{array}$ & \begin{tabular}{|l}
11.079 \\
$\mathbf{0 . 0 4 9}$ \\
$* 0.136$
\end{tabular} & $\begin{array}{l}19.935 \\
0.001 \\
* 0.182\end{array}$ & $\begin{array}{l}6.843 \\
0.232 \\
-\end{array}$ & $\begin{array}{l}28.581 \\
0.000 \\
* 0.218\end{array}$ & $\begin{array}{l}6.255 \\
0.282 \\
-\end{array}$ & $\begin{array}{l}5.393 \\
0.369 \\
-\end{array}$ & $\begin{array}{l}4.608 \\
0.465 \\
-\end{array}$ \\
\hline
\end{tabular}




\begin{tabular}{|c|c|c|c|c|c|c|c|c|}
\hline Variable & $\begin{array}{l}\mathrm{Chi}^{2} \\
p \\
* \mathrm{~V} / \\
* * \mathrm{Fi}\end{array}$ & Diabetes & $\begin{array}{l}\text { Hyperten- } \\
\text { sion }\end{array}$ & $\begin{array}{l}\text { Asthma/ } \\
\text { /COPD }\end{array}$ & $\begin{array}{l}\text { Atheroscle- } \\
\text { rosis }\end{array}$ & $\begin{array}{l}\text { Other car- } \\
\text { diovascular } \\
\text { diseases }\end{array}$ & $\begin{array}{l}\text { Hyperthy- } \\
\text { roidism }\end{array}$ & $\begin{array}{l}\text { Digestive } \\
\text { diseases }\end{array}$ \\
\hline $\begin{array}{l}\text { Years worked } \\
\text { one year or less } \\
1-3 \text { years } \\
3-8 \text { years } \\
8-18 \text { years } \\
\text { over } 18 \text { years }\end{array}$ & $\begin{array}{l}\mathrm{Chi}^{2} \\
p \\
* \mathrm{~V} / \\
* * \mathrm{Fi}\end{array}$ & $\begin{array}{l}3.338 \\
0.502 \\
-\end{array}$ & $\begin{array}{l}40.907 \\
0.000 \\
* 0.321\end{array}$ & $\begin{array}{l}4.392 \\
0.3555 \\
-\end{array}$ & $\begin{array}{l}8.461 \\
0.076 \\
-\end{array}$ & \begin{tabular}{|l|}
12.882 \\
$\mathbf{0 . 0 1 1}$ \\
$* 0.180$
\end{tabular} & $\begin{array}{l}1.988 \\
0.737 \\
-\end{array}$ & $\begin{array}{l}1.199 \\
0.878 \\
-\end{array}$ \\
\hline $\begin{array}{l}\text { Shift work } \\
\text { yes } \\
\text { no }\end{array}$ & $\begin{array}{l}\mathrm{Chi}^{2} \\
p \\
* \mathrm{~V} / \\
* * \mathrm{Fi} \\
\end{array}$ & $\begin{array}{l}0.436 \\
0.508 \\
-\end{array}$ & $\begin{array}{l}0.370 \\
0.542 \\
-\end{array}$ & $\begin{array}{l}0.000 \\
0.992 \\
-\end{array}$ & $\begin{array}{l}0.443 \\
0.505 \\
-\end{array}$ & $\begin{array}{l}1.358 \\
0.243 \\
-\end{array}$ & $\begin{array}{l}0.000 \\
0.998 \\
-\end{array}$ & $\begin{array}{l}0.382 \\
0.536 \\
-\end{array}$ \\
\hline $\begin{array}{l}\text { Self-assessed finan- } \\
\text { cial standing } \\
\text { very good } \\
\text { good } \\
\text { satisfactory } \\
\text { bad }\end{array}$ & $\begin{array}{l}\mathrm{Chi}^{2} \\
p \\
* \mathrm{~V} / \\
* * \mathrm{Fi}\end{array}$ & \begin{tabular}{|l}
10.465 \\
$\mathbf{0 . 0 1 5}$ \\
$* 0.132$
\end{tabular} & $\begin{array}{l}6.943 \\
0.073 \\
-\end{array}$ & $\begin{array}{l}12.252 \\
\mathbf{0 . 0 0 6} \\
* 0.143\end{array}$ & \begin{tabular}{|l|}
14.265 \\
$\mathbf{0 . 0 0 2}$ \\
$* 0.154$
\end{tabular} & $\begin{array}{l}8.830 \\
\mathbf{0 . 0 3 1} \\
*_{0} 0.121\end{array}$ & $\begin{array}{l}10.653 \\
\mathbf{0 . 0 1 3} \\
* 0.133\end{array}$ & $\begin{array}{l}5.497 \\
0.138 \\
-\end{array}$ \\
\hline
\end{tabular}

$\mathrm{V}$ - Cramer's V coefficient; ** Fi - Fi coefficient.

Hypothesis 2: Insomnia is often related to chronic diseases

We analysed the relationship between insomnia and chronic diseases reported by the respondents. A statistically significant relationship was observed between insomnia and hypertension $(p \leq 0.001)$ - every fourth person with hypertension suffered from insomnia ( $n=30 ; 25.86 \%$ ) and had chronic diseases not included in the questionnaire $(p \leq 0.001)$ (Table 4).

The largest group with insomnia included those who had chronic diseases not mentioned in the questionnaire $(p=$ 0.009). Insomnia was observed in 30 individuals (8.02\%), and borderline insomnia in 68 subjects (25.76\%) (Table 5).

In the group of unemployed subjects $(n=396)$, insomnia was statistically significantly related to diseases other than those mentioned above $(p=0.037)$ (Table 6).

\begin{tabular}{|c|c|c|c|c|c|}
\hline \multirow{2}{*}{ Chronic diseases } & \multicolumn{3}{|c|}{ Athens Insomnia Scale (AIS) } & \multirow[b]{2}{*}{$\mathrm{Chi}^{2}$} & \multirow[b]{2}{*}{$\begin{array}{l}p \\
* V\end{array}$} \\
\hline & $\begin{array}{l}\text { no insomnia } \\
n(\%)\end{array}$ & $\begin{array}{l}\text { borderline insomnia } \\
n(\%)\end{array}$ & $\begin{array}{l}\text { insomnia } \\
n(\%)\end{array}$ & & \\
\hline $\begin{array}{l}\text { Diabetes } \\
\text { yes } \\
\text { no }\end{array}$ & $\begin{array}{l}14(33.33) \\
287(51.71)\end{array}$ & $\begin{array}{l}17(40.48) \\
173(31.17)\end{array}$ & $\begin{array}{l}11(26.19) \\
95(17.12)\end{array}$ & 5.488 & 0.064 \\
\hline $\begin{array}{l}\text { Hypertension } \\
\text { yes } \\
\text { no }\end{array}$ & $\begin{array}{l}38(32.76) \\
263(54.68)\end{array}$ & $\begin{array}{l}48(41.38) \\
142(29.52)\end{array}$ & $\begin{array}{l}30(25.86) \\
76(15.8)\end{array}$ & 18.363 & $\begin{array}{l}\leq 0.001 \\
* 0.175\end{array}$ \\
\hline $\begin{array}{l}\text { Asthma/COPD } \\
\text { yes } \\
\text { no }\end{array}$ & $\begin{array}{l}13(36.11) \\
287(51.25)\end{array}$ & $\begin{array}{l}15(41.67) \\
175(31.25)\end{array}$ & $\begin{array}{l}8(22.22) \\
98(17.5)\end{array}$ & 3.115 & 0.210 \\
\hline $\begin{array}{l}\text { Atherosclerosis } \\
\text { yes } \\
\text { no }\end{array}$ & $\begin{array}{l}7(31.82) \\
294(51.13)\end{array}$ & $\begin{array}{l}8(36.36) \\
182(31.65)\end{array}$ & $\begin{array}{l}7(31.82) \\
99(17.22)\end{array}$ & 4.259 & 0.118 \\
\hline $\begin{array}{l}\text { Other cardiovascular diseases } \\
\text { yes } \\
\text { no }\end{array}$ & $\begin{array}{l}7(30.43) \\
294(51.22)\end{array}$ & $\begin{array}{l}11(47.83) \\
179(31.18)\end{array}$ & $\begin{array}{l}5(21.74) \\
101(17.6)\end{array}$ & 4.032 & 0.133 \\
\hline $\begin{array}{l}\text { Hyperthyroidism } \\
\text { yes } \\
\text { no } \\
\end{array}$ & \begin{tabular}{|l|}
$11(29.73)$ \\
$290(51.79)$ \\
\end{tabular} & $\begin{array}{l}13(35.14) \\
177(31.61) \\
\end{array}$ & $\begin{array}{l}13(35.14) \\
93(16.61) \\
\end{array}$ & 10.194 & 0.006 \\
\hline $\begin{array}{l}\text { Digestive diseases } \\
\text { yes } \\
\text { no }\end{array}$ & $\begin{array}{l}17(48.57) \\
284(50.53)\end{array}$ & $\begin{array}{l}13(37.14) \\
177(31.49)\end{array}$ & $\begin{array}{l}5(14.29) \\
101(17.97)\end{array}$ & 0.607 & 0.738 \\
\hline $\begin{array}{l}\text { Other diseases } \\
\text { yes } \\
\text { no }\end{array}$ & \begin{tabular}{|l}
$215(57.33)$ \\
$86(38.74)$
\end{tabular} & $\begin{array}{l}104(27.73) \\
86(38.74)\end{array}$ & $\begin{array}{l}56(14.93) \\
50(22.52)\end{array}$ & 19.393 & $\begin{array}{l}\leq 0.001 \\
* 0.180\end{array}$ \\
\hline
\end{tabular}

$* \mathrm{~V}$-Cramer's $\mathrm{V}$ coefficient. 


\begin{tabular}{|c|c|c|c|c|c|}
\hline \multirow[t]{2}{*}{ Chronic diseases } & \multicolumn{3}{|c|}{ Athens Insomnia Scale (AIS) } & \multirow[t]{2}{*}{$\mathrm{Chi}^{2}$} & \multirow{2}{*}{$\begin{array}{l}p \\
* V\end{array}$} \\
\hline & $\begin{array}{l}\text { no insomnia } \\
n(\%)\end{array}$ & $\begin{array}{l}\text { borderline insomnia } \\
n(\%)\end{array}$ & $\begin{array}{l}\text { insomnia } \\
n(\%)\end{array}$ & & \\
\hline $\begin{array}{l}\text { Diabetes } \\
\text { yes } \\
\text { no }\end{array}$ & $\begin{array}{l}10(52.63) \\
218(57.82)\end{array}$ & $\begin{array}{l}5(26.32) \\
110(29.18)\end{array}$ & $\begin{array}{l}4(21.05) \\
49(13)\end{array}$ & 1.012 & 0.602 \\
\hline $\begin{array}{l}\text { Hypertension } \\
\text { yes } \\
\text { no }\end{array}$ & $\begin{array}{l}23(39.66) \\
205(60.65)\end{array}$ & $\begin{array}{l}22(37.93) \\
93(27.51)\end{array}$ & $\begin{array}{l}13(22.41) \\
40(11.83)\end{array}$ & 9.779 & $\begin{array}{l}\mathbf{0 . 0 0 7} \\
*_{0} 0.157 \\
\end{array}$ \\
\hline $\begin{array}{l}\text { Asthma/COPD } \\
\text { yes } \\
\text { no }\end{array}$ & $\begin{array}{l}9(52.94) \\
218(57.67) \\
\end{array}$ & $\begin{array}{l}4(23.53) \\
111(29.37)\end{array}$ & $\begin{array}{l}4(23.53) \\
49(12.96) \\
\end{array}$ & 1.607 & 0.447 \\
\hline $\begin{array}{l}\text { Atherosclerosis } \\
\text { yes } \\
\text { no }\end{array}$ & $\begin{array}{l}1(33.33) \\
227(57.76)\end{array}$ & $\begin{array}{l}- \\
115(29.26)\end{array}$ & $\begin{array}{l}2(66.67) \\
51(12.98)\end{array}$ & 7.598 & $\begin{array}{l}0.022 \\
*_{0} 0.138\end{array}$ \\
\hline $\begin{array}{l}\text { Other cardiovascular diseases } \\
\text { yes } \\
\text { no }\end{array}$ & $\begin{array}{l}4(40) \\
224(58.03)\end{array}$ & $\begin{array}{l}5(50) \\
110(28.5)\end{array}$ & $\begin{array}{l}1(10) \\
52(13.47)\end{array}$ & 2.190 & 0.334 \\
\hline $\begin{array}{l}\text { Hyperthyroidism } \\
\text { yes } \\
\text { no } \\
\end{array}$ & $\begin{array}{l}9(36) \\
219(59.03)\end{array}$ & $\begin{array}{l}8(32) \\
107(28.84) \\
\end{array}$ & $\begin{array}{l}8(32) \\
45(12.13)\end{array}$ & 9.147 & $\begin{array}{l}\mathbf{0 . 0 1 0} \\
*_{0} 0.151 \\
\end{array}$ \\
\hline $\begin{array}{l}\text { Digestive diseases } \\
\text { yes } \\
\text { no }\end{array}$ & $\begin{array}{l}12(54.55) \\
216(57.75)\end{array}$ & $\begin{array}{l}9(40.9) \\
106(28.34)\end{array}$ & $\begin{array}{l}1(4.55) \\
52(13.9)\end{array}$ & 2.526 & 0.282 \\
\hline $\begin{array}{l}\text { Other diseases } \\
\text { yes } \\
\text { no }\end{array}$ & $\begin{array}{l}166(62.88) \\
62(46.97)\end{array}$ & $\begin{array}{l}68(25.76) \\
47(35.61)\end{array}$ & $\begin{array}{l}30(8.02) \\
23(17.42)\end{array}$ & 9.222 & $\begin{array}{l}0.009 \\
*^{0} 0.152\end{array}$ \\
\hline
\end{tabular}

$* \mathrm{~V}$-Cramer's $\mathrm{V}$ coefficient.

\begin{tabular}{|c|c|c|c|c|c|}
\hline \multirow[t]{2}{*}{ Chronic diseases } & \multicolumn{3}{|c|}{ Athens Insomnia Scale (AIS) } & \multirow[t]{2}{*}{$\mathrm{Chi}^{2}$} & \multirow{2}{*}{$\begin{array}{l}p \\
* V\end{array}$} \\
\hline & $\begin{array}{l}\text { no insomnia } \\
n(\%)\end{array}$ & $\begin{array}{l}\text { borderline insomnia } \\
n(\%)\end{array}$ & $\begin{array}{l}\text { insomnia } \\
n(\%)\end{array}$ & & \\
\hline $\begin{array}{l}\text { Diabetes } \\
\text { yes } \\
\text { no }\end{array}$ & $\begin{array}{l}4(17.39) \\
69(38.76)\end{array}$ & $\begin{array}{l}12(52.17) \\
63(35.39)\end{array}$ & $\begin{array}{l}7(30.43) \\
46(25.84)\end{array}$ & 4.261 & 0.118 \\
\hline $\begin{array}{l}\text { Hypertension } \\
\text { yes } \\
\text { no }\end{array}$ & $\begin{array}{l}15(25.86) \\
58(40.56)\end{array}$ & $\begin{array}{l}26(44.83) \\
49(34.27)\end{array}$ & $\begin{array}{l}17(29.31) \\
36(25.17)\end{array}$ & 3.955 & 0.138 \\
\hline $\begin{array}{l}\text { Asthma/COPD } \\
\text { yes } \\
\text { no }\end{array}$ & $\begin{array}{l}4(21.05) \\
69(37.91)\end{array}$ & $\begin{array}{l}11(57.89) \\
64(35.16)\end{array}$ & $\begin{array}{l}4(21.05) \\
49(26.92)\end{array}$ & 3.953 & 0.138 \\
\hline $\begin{array}{l}\text { Atherosclerosis } \\
\text { yes } \\
\text { no }\end{array}$ & $\begin{array}{l}6(31.58) \\
67(36.81)\end{array}$ & $\begin{array}{l}8(42.11) \\
67(36.81)\end{array}$ & $\begin{array}{l}5(26.32) \\
48(26.37)\end{array}$ & 0.258 & 0.878 \\
\hline $\begin{array}{l}\text { Other cardiovascular diseases } \\
\text { yes } \\
\text { no }\end{array}$ & $\begin{array}{l}3(23.08) \\
70(37.23)\end{array}$ & $\begin{array}{l}6(46.15) \\
69(36.7)\end{array}$ & $\begin{array}{l}4(30.77) \\
49(26.92)\end{array}$ & 1.064 & 0.587 \\
\hline $\begin{array}{l}\text { Hyperthyroidism } \\
\text { yes } \\
\text { no }\end{array}$ & $\begin{array}{l}2(16.67) \\
71(37.57)\end{array}$ & $\begin{array}{l}5(41.67) \\
70(37.04) \\
\end{array}$ & $\begin{array}{l}5(41.67) \\
48(25.4) \\
\end{array}$ & 2.554 & 0.278 \\
\hline $\begin{array}{l}\text { Digestive diseases } \\
\text { yes } \\
\text { no }\end{array}$ & $\begin{array}{l}5(38.46) \\
68(36.17)\end{array}$ & $\begin{array}{l}4(30.77) \\
71(37.77)\end{array}$ & $\begin{array}{l}4(30.77) \\
49(26.06)\end{array}$ & 0.279 & 0.869 \\
\hline $\begin{array}{l}\text { Other diseases } \\
\text { yes } \\
\text { no }\end{array}$ & $\begin{array}{l}49 \text { (44.14) } \\
24(26.67)\end{array}$ & $\begin{array}{l}36(32.43) \\
39(43.33)\end{array}$ & $\begin{array}{l}26(23.42) \\
27(30)\end{array}$ & 6.578 & $\begin{array}{l}\mathbf{0 . 0 3 7} \\
* 0.180\end{array}$ \\
\hline
\end{tabular}

\footnotetext{
$* \mathrm{~V}$-Cramer's $\mathrm{V}$ coefficient.
} 


\begin{tabular}{|c|c|c|c|c|c|c|}
\hline \multirow[t]{2}{*}{ Variable } & \multicolumn{3}{|c|}{ Athens Insomnia Scale (AIS) } & \multirow[t]{2}{*}{$\mathrm{Chi}^{2}$} & \multirow[t]{2}{*}{$p$} & \multirow[t]{2}{*}{$* \mathbf{V}$} \\
\hline & $\begin{array}{l}\text { no insomnia } \\
n(\%)\end{array}$ & $\begin{array}{l}\text { borderline } \\
\text { insomnia } \\
n(\%)\end{array}$ & $\begin{array}{l}\text { insomnia } \\
n(\%)\end{array}$ & & & \\
\hline $\begin{array}{l}\text { Employment status } \\
\text { employed } \\
\text { unemployed }\end{array}$ & $\begin{array}{l}228(57.58) \\
73(36.32) \\
\end{array}$ & $\begin{array}{l}115 \text { (29.04) } \\
75(37.31) \\
\end{array}$ & $\begin{array}{l}53(13.38) \\
53(26.37) \\
\end{array}$ & 27.476 & $\leq 0.001$ & $* 0.214$ \\
\hline $\begin{array}{l}\text { Shift work } \\
\text { yes } \\
\text { no }\end{array}$ & \begin{tabular}{|l}
$95(47.26)$ \\
$200(51.95)$ \\
\end{tabular} & \begin{tabular}{|l|}
$66(32.84)$ \\
$123(31.95)$ \\
\end{tabular} & \begin{tabular}{|l}
$40(19.9)$ \\
$62(16.1)$ \\
\end{tabular} & 1.701 & 0.427 & - \\
\hline $\begin{array}{l}\text { Form of employment } \\
\text { employment contract } \\
\text { freelance agreement } \\
\text { contract for a specific task } \\
\text { contract } \\
\text { other }\end{array}$ & $\begin{array}{l}162(58.48) \\
36(49.32) \\
- \\
12(60) \\
25(73.53) \\
\end{array}$ & $\begin{array}{l}80(28.88) \\
24(32.88) \\
2(40) \\
6(30) \\
7(20.59) \\
\end{array}$ & $\begin{array}{l}35(12.63) \\
13(17.81) \\
3(60) \\
2(10) \\
2(5.88) \\
\end{array}$ & $\begin{array}{l}2.083 \\
1.958 \\
10.792 \\
0.281 \\
4.861 \\
\end{array}$ & $\begin{array}{l}0.352 \\
0.375 \\
\mathbf{0 . 0 0 4} \\
0.868 \\
0.087 \\
\end{array}$ & $\begin{array}{l}- \\
- \\
* 0.160 \\
- \\
-\end{array}$ \\
\hline $\begin{array}{l}\text { Years worked } \\
\text { one year or less } \\
1-3 \text { years } \\
3-8 \text { years } \\
8-18 \text { years } \\
\text { over } 18 \text { years } \\
\end{array}$ & $\begin{array}{l}57(55.34) \\
64(69.57) \\
46(54.76) \\
31(48.44) \\
30(56.6) \\
\end{array}$ & \begin{tabular}{|l|}
$34(33.01)$ \\
$20(21.74)$ \\
$23(27.38)$ \\
$23(35.94)$ \\
$15(28.3)$ \\
\end{tabular} & $\begin{array}{l}12(11.65) \\
8(8.7) \\
15(17.86) \\
10(15.62) \\
8(15.09) \\
\end{array}$ & 10.178 & 0.252 & - \\
\hline $\begin{array}{l}\text { Self-assessed financial standing } \\
\text { very good } \\
\text { good } \\
\text { satisfactory } \\
\text { bad }\end{array}$ & $\begin{array}{l}42(67.74) \\
165(55.18) \\
90(43.27) \\
4(14.29)\end{array}$ & $\begin{array}{l}11(17.74) \\
87(29.1) \\
82(39.42) \\
10(35.71)\end{array}$ & $\begin{array}{l}9(14.52) \\
47(15.72) \\
36(17.31) \\
14(50)\end{array}$ & 40.349 & $\leq 0.001$ & $* 0.183$ \\
\hline
\end{tabular}

$* \mathrm{~V}$-Cramer's $\mathrm{V}$ coefficient.

\section{Hypothesis 3: Sleep problems are related to em- ployment status}

Insomnia was significantly more common among unemployed subjects $(p \leq 0.001)$. A statistical relationship was demonstrated between insomnia and work under a contract for a specific task $(p=0.004)$ (Table 7).

\section{Discussion}

It was found in our analysis that people in the non-working group more often had borderline insomnia ( $n=75 ; 37.31 \%$ ). A study of over forty thousand respondents conducted by Vancampfort et al. demonstrated that people having sedentary jobs and lifestyles (8-11 hours per day) were at a 1.75 times higher risk (95\% Cl: 1.21-2.40) of developing sleep disorders than those who spent less than four hours per day in a sedentary life [12]. Based on these results, it can be assumed that insomnia is not only a matter of employment status, but also concerns lifestyle and physical activity.

We observed insomnia in $19.9 \%$ and borderline insomnia in $32.84 \%$ of shift workers. The association between shift work and insomnia was also confirmed by Vallières et al., who reported that night-shift workers had worse quality of sleep than those working day shifts. In addition, night-shift workers were overexcited and needed more time to fall asleep [13]. This corresponds with the results of another study, in which insomnia was more common among people who started shifts in the middle of the night [14].

Our investigation also revealed a statistically significant relationship between insomnia and work under a contract for a specific task - three out of five subjects declaring this form of employment led to insomnia $(p=0.004)$. Żołnierczyk-Zreda, who reviewed literature concerning the impact of the form of an employment contract on employees' health and professional functioning, concluded that lack of permanent employment leads to overall deterioration of mental and physical health and, consequently, to sleep problems [15].

In our study, insomnia was noted in over $26.19 \%$ of respondents with diabetes; however, it was more common among those unemployed (30.43\%). The relationship between these variables has been confirmed by many studies. In 2018, Hein et al. conducted a study on 1,300 individuals with and without type 2 diabetes, finding that insomnia sufferers with type 2 diabetes had a shorter duration of sleep, and their sleep was less regenerative [16].

Another important contributor to insomnia is hypertension. We noted a statistically significant relationship $(p \leq 0.001)$ between hypertension and insomnia, both in the whole study sample and in the subgroup of employed respondents ( $p=$ 0.007). According to Vgontzas et al., insomnia entails a substantially higher risk of increased blood pressure. These authors claimed that the co-existence of insomnia and short sleep duration (less than 5 hours) raised the risk of hypertension by about $500 \%(\mathrm{OR}=5.12,95 \% \mathrm{Cl}: 2.2-11.8)$ compared with the group without insomnia and with a sleep duration $>6 \mathrm{~h}$ [5]. Based on their study of over 1,000 people, Błaszczyk et al. informed that the most numerous subjects in the group with severe insomnia were those with third-degree hypertension $(77.3 \% ; p \leq 0.001)$ [17].

Although our study did not demonstrate any association between asthma and insomnia, such a relationship has been reported by other researchers [18-20]. Patients with asthma were also found to have poorer quality of sleep than the control group [19]. The fact that asthma symptoms exacerbate at night may explain the link between these two disorders [19].

In our study, hyperthyroidism was statistically significantly related to gender $(p=0.000)$ - it was considerably more common among women (8.89\%). Caputo et al. conducted a study of $4,400,000$ residents of Piedmont in Italy. They found that in 2012-2018, 33,257 people were diagnosed with hyperthyroidism, including 9,165 men and 24,092 women. The general inci- 
dence rate was 756 per 100,000 residents (95\% Cl: 748-764). A detailed analysis with regard to gender showed that both the incidence and prevalence rates were higher for women than for men - the incidence rate for women was 2.18 (95\% Cl: 2.10 $-2.26)$, and the prevalence rate was 2.77 (95\% Cl: 2.71-2.82) [20]. Considering that Caputo et al. carried out their research in a mountain terrain, these results might have been associated with the lower level of iodine in the air.

\section{Limitations of the study}

- The majority of unemployed individuals were in the age bracket of over 56 years, which might have had an effect on the incidence of chronic diseases.

- The study sample included more employed individuals.

\section{Conclusions}

1. Diseases were more common in unemployed respondents, which might have been due to the fact that they were mainly people over 56 years of age.

2. Insomnia itself can be the cause of the onset or deepening of diseases. Insomnia and chronic diseases, in many respects, are a consequence of lifestyle and hypokinesia.

3. The age factor and working and living conditions (contract work, shift work, unemployment) are factors contributing to the occurrence or worsening of insomnia and chronic diseases. Insomnia and a lack of depth and quality of sleep can be due to the level of professional and life stress, working and living conditions, social instability and chronic diseases.

Source of funding: This work was funded from the authors' own resources. Conflicts of interest: The authors declare no conflicts of interest.

\section{References}

1. Szelenberger W, Jakitowicz J. Bezsenność - problem diagnostyczny i terapeutyczny. In: Szelenberger W, Nowicki Z, eds. Zaburzenia snu. Diagnostyka i leczenie, wybrane zagadnienia. Kraków: Komitet Redakcyjno-Wydawniczy Polskiego Towarzystwa Psychiatrycznego; 1999: 19-31 (in Polish).

2. American Psychiatric Association. Diagnostic and Statistical Manual of Mental Disorders. 5th ed. Washington: American Psychiatric Association; 2013.

3. Stavropoulos K, Imprialos KP, Doumas M, et al. Insomnia and hypertension: a misty landscape. J Clin Hypertens (Greenwich) 2019; 21(6): 835-837, doi: 10.1111/jch.13519.

4. Liu RQ, Qian Z, Trevathan E, et al. Poor sleep quality associated with high risk of hypertension and elevated blood pressure in China: results from a large population-based study. Hypertens Res 2016; 39(1): 54-59.

5. Vgontzas AN, Liao D, Bixler EO, et al. Insomnia with objective short sleep duration is associated with a high risk for hypertension. Sleep 2009; 32(4): 491-497.

6. Lemmer B, Oster $\mathrm{H}$. The role of circadian rhythms in the hypertension of diabetes mellitus and the metabolic syndrome. Curr Hypertens Rep 2018; 20: 43, doi: 10.1007/s11906-018-0843-5.

7. Pallayova M, Donic V, Gresova S, et al. Do differences in sleep architecture exist between persons with type 2 diabetes and nondiabetic controls? J Diabetes Sci Technol 2010; 4: 344-352.

8. Tsujimura T, Matsuo Y, Keyaki T, et al. Correlations of sleep disturbance with the immune system in type 2 diabetes mellitus. Diabetes Res Clin Pract 2009; 85: 286-292.

9. Xia L, Chen GH, Li ZH, et al. Alterations in hypothalamus-pituitary-adrenal/thyroid axes and gonadotropin-releasing hormone in the patients with primary insomnia: a clinical research. PLOS ONE 2013; 8(8): e71065, doi: 10.1371/journal.pone.0071065.

10. Ikegami K, Refetoff S, Van Cauter E, et al. Interconnection between circadian clocks and thyroid function. Nat Rev Endocrinol 2019; 15(10): 590-600.

11. Masek K, Slánský J, Petrovický P, et al. Neuroendocrine immune interactions in health and disease. Int Immunopharmacol 2003; 3(8): $1235-1246$.

12. Vancampfort D, Stubbs B, Firth J, et al. Sedentary behaviour and sleep problems among 42,489 community-dwelling adults in six lowand middle-income countries. J Sleep Res 2018: e12714, doi: 10.1111/jsr.12714.

13. Vallières A, Azaiez A, Moreau V, et al. Insomnia in shift work. Sleep Med 2014; 15(12): 1440-1448.

14. Pepin E, Gillet P, Sauvet F, et al. Shift work, night work and sleep disorders among pastry cookers and shopkeepers in France: a crosssectional survey. BMJ Open 2018; 8(5), doi: 10.1136/bmjopen-2017-019098.

15. Żołnierczyk-Zreda D. Rodzaj umowy o pracę a zdrowie i funkcjonowanie zawodowe pracowników - przegląd badań. Med Pr 2015; 66(4): 565-573 (in Polish).

16. Hein M, Lanquart JP, Loas G, et al. Prevalence and risk factors of type 2 diabetes in insomnia sufferers: a study on 1311 individuals referred for sleep examinations. Sleep Med 2018; 46: 37-45.

17. Błaszczyk R, Wysokiński A, Ciota $\mathrm{M}$, et al. Zaburzenia snu oraz senność dzienna u pacjentów z nadciśnieniem tętniczym. Pol Przegl Kardiol 2010; 12(2): 109-115 (in Polish).

18. Wolińska W, Pawlak IE, Mroczek B. Coexistence of insomnia and chronic diseases in over 60 years old. Fam Med Primary Care Rev 2016 ; 18(3): 364-367.

19. Sundbom F, Lindberg E, Bjerg A, et al. Asthma symptoms and nasal congestion as independent risk factors for insomnia in a general population: results from the GA(2)LEN survey. Allergy 2013; 68(2): 213-219.

20. Caputo M, Pecere A, Sarro A, et al. Incidence and prevalence of hyperthyroidism: a population-based study in the Piedmont Region, Italy. Endocrine 2020, doi: 10.1007/s12020-020-02222-7.

Tables: 7

Figures: 0

References: 20

Received: 16.04 .2020

Reviewed: 02.05.2020

Accepted: 04.06 .2020
Address for correspondence

Weronika Wolińska, PhD

Zakład Nauk Humanistycznych w Medycynie

Pomorski Uniwersytet Medyczny w Szczecinie

ul. Gen. D. Chłapowskiego 11

70-193 Szczecin

Polska

Tel.: +48 508 301-892

E-mail: weronika.wolinska@pum.edu.pl 